\title{
Reduction of global interference in functional multidistance near-infrared spectroscopy using empirical mode decomposition and recursive least squares: a Monte Carlo study
}

\author{
Y. Zhang \\ zyhit@hit.edu.cn \\ J. Sun \\ P. Rolfe \\ peterrolfe@aol.com
}

\author{
Dept. Automatic Meas. \& Cont., Harbin Institute of Technology, No.92, West Da-zhi Street, Nangang \\ District, Harbin 150001, China \\ Dept. Automatic Meas. \& Cont., Harbin Institute of Technology, No.92, West Da-zhi Street, Nangang \\ District, Harbin 150001, China \\ Dept. Automatic Meas. \& Cont., Harbin Institute of Technology, No.92, West Da-zhi Street, Nangang \\ District, Harbin 150001, China
}

Functional near-infrared spectroscopy (fNIRS) is a sensitive technique that has the potential to detect haemodynamic changes during the performance of specific activation tasks. However, in real situations, fNIRS recordings are often corrupted by physiological phenomena, especially by cardiac contraction, breathing and blood pressure fluctuations, and these forms of interference can severely limit the utility of fNIRS. We present a novel fNIRS enhancement based on the multidistance fNIRS method with short-distance and long-distance optode pairs. With this method empirical mode decomposition (EMD) is applied to decompose the short-distance fNIRS measurement into a series of intrinsic mode functions (IMFs). By utilizing the weighting coefficients for the IMFs, we derive an estimate for global interference in the long-distance fNIRS measurements. We recover the evoked brain activity by minimizing least squares between the long-distance measurements and the estimated global interference. To accelerate the computation we adopt the recursive least squares (RLS) to decrease the computation complexity due to the matrix inversion. Monte Carlo simulations of photon propagation through a five-layered slab model of a human adult head were implemented to evaluate our methodology. The results demonstrate that the EMD-RLS method can effectively remove contamination from the evoked brain activity. [DOI: 10.2971/jeos.2011.11033]

Keywords: functional near-infrared spectroscopy, interference, multidistance measurement, empirical mode decomposition, recursive least squares

\section{INTRODUCTION}

Near-infrared spectroscopy (NIRS) has been developed to allow noninvasive measurement of optically absorbing and scattering molecules in medicine and biology [1]. Although NIRS may be useful in a variety of tissues and organs there has been a major interest in its use for studies of the brain and it has been applied in adults [2], the fetus [3] and the newborn [4]. Of particular importance has been the ability of the method to monitor changes in cerebral oxyhaemoglobin and deoxyhaemoglobin $\left(\mathrm{HbO}_{2}, \mathrm{HHb}\right)$, total haemoglobin $\left(\mathrm{Hb}_{\text {tot }}\right)$ and the redox state of the respiratory enzyme cytochrome oxidase $\left(\mathrm{cyt}_{\mathrm{aa} 3}\right)[5,6]$. The quantitative measurement of the target chromophores in terms of absolute concentrations has always been a challenge and this has directed NIRS research, over several decades, particularly with respect to instrumentation and in the underlying theoretical considerations. This has led to the use of continuous wave, time-resolved, intensity modulated, and spatially-resolved instruments [7] based on theories of photon propagation through tissue described by the Radiative Transport Equation, the diffusion approximation and diffuse reflectance [8].

The use of stimuli to evoke physiological responses has been one particular area of relevance to NIRS, leading to what has become the well-recognised method of functional nearinfrared spectroscopy (fNIRS). This has been demonstrated to be able to determine cerebral concentration changes of $\mathrm{HbO}_{2}$ and $\mathrm{HHb}$ during functional activation of the cerebral cortex [9]. fNIRS may be compared with other techniques, such as functional magnetic resonance imaging (fMRI), magnetoencephalography (MEG), positron emission tomography (PET), and electroencephalography (EEG) $[10,11]$. It does appear to have several advantages over these other methods, such as portability, fewer physical restrictions and greater practicality, good temporal resolution, safety, and inexpensive instrumentation. In fNIRS studies, measurement of the concentration changes of $\mathrm{HbO}_{2}$ and $\mathrm{HHb}$, the two primary absorbing chromophores in the brain tissue that vary dynamically with a functional task, is achieved. This then provides a useful description of the cerebral haemodynamics. However, there are problems in using fNIRS due to the presence of physiological interference, which is mainly from perturbations caused by cardiac events, such as changes in blood flow, blood pressure and blood volume following cardiac contractions, as well as from breathing [5]. All of these forms of interference are located both in the vasculature of the superficial layer of the brain and inside the brain, and are generally termed "global interference" or "systemic physiological interference". Severe contamination of global interference remains a serious problem for fNIRS interpretation and analysis. This has meant that 
without appropriate global interference reduction the full potential of fNIRS has not yet been realised.

Previous attempts have been made to suppress global interference and thereby improve the brain activity measurements. The use of low pass filtering techniques to remove the interference caused by cardiac oscillations has been reported [12]. However, low pass filtering is not effective at removing the specific physiological noise signals such as respiratory and blood pressure variation since these fluctuations are difficult to be distinguished from the haemodynamic response to brain activity by frequency characteristics alone. Saager et al. [13] utilized a dual-detector system and least squares method to remove top-layer-only fluctuations and validated the effect of the methodology by performing Monte Carlo simulations based on a two-layer turbid media model. Zhang et al. [11] proposed a spatial eigenfiltering algorithm to separate an activity-evoked response from systemic physiological interference in diffuse optical imaging data. They assumed that the first several spatial eigenvectors of the baseline concentration correlation matrices were dominated by interference patterns and hold most of the total energy. Morren et al. [14] and Zhang et al. [15] adopted adaptive filtering to remove global interference. The difference between the two approaches is that the former used signals from a pulse oximeter as a reference and the latter used signals from short interoptode distance as a reference. A Kalman filtering model has also been used to analyze interference components $[16,17]$. Abdelnour et al. proposed an adaptive general linear model based on a Kalman filtering algorithm for real-time assessment of brain function [18]. Despite the fact that the general linear model in their papers exhibited the potential for removal of physiological signals from cardiac, respiratory, and Mayer wave fluctuations, the authors expressly indicated that prior distributions must be assumed for both the process and observed noise in the Kalman filtering model.

Empirical mode decomposition (EMD) and Hilbert spectral analysis (HSA) have been shown to be able to separate, identify and remove interference arising from some cardiac events and breathing [19]. This technique has the advantage of simplicity in instrument design because it only needs one source and one detector in the NIRS probe. However, the EMD-HSA technique may not be appropriate for removing other physiological fluctuations such as blood pressure and other lower frequency variations, although these fluctuations in superficial layers are highly correlated with those in deep layers.

In our study presented here we adopt the spatially-resolved or multidistance measurement method and a theoretical analysis of global interference reduction based on EMD and the least squares criterion. The short-distance fNIRS measurement is treated as the reference channel comprising of superficial haemodynamic changes induced by physiological fluctuations and the long-distance fNIRS measurement is treated as the signal channel containing both the functional haemodynamic response and global interference. We aim to remove global interference that is correlated with superficial haemodynamic fluctuations, evoked by cardiac contractions, breathing, blood pressure, etc. By decomposing the short-distance measurement with the EMD algorithm, we separated the in- terference into different intrinsic mode functions (IMFs) possessing distinct frequency characteristics. The least squares criterion was then used to adjust the corresponding weighting coefficients to estimate global interference with the obtained IMFs. To accelerate the computation we adopted the recursive least squares (RLS) to decrease the computation complexity. Monte Carlo simulations of a five-layer human model were used to investigate the performance of the EMD-RLS for removing global interference in brain activity measurement.

\section{Theory}

\subsection{Empirical Mode Decomposition}

EMD is an adaptive signal processing technique, which aims at extracting all the fundamental modes embedded in a signal without any requirement of stationarity or linearity of the data [20]. EMD is based on an iterative algorithm in which the highest frequency oscillation is removed from the data with each repetition. Following each iteration a residue remains containing lower frequency information. The process is repeated until only a trend remains, leading to a local, adaptive decomposition, in which intrinsic oscillations within the data may be identified.

EMD is derived from the simple assumption that any signal consists of different coexisting modes of oscillation with a separated time-scale. Each of these oscillatory modes is represented by an intrinsic mode function (IMF) and the IMF must satisfy two criteria. Firstly, the number of extrema and the number of zero-crossings must either equal or differ at most by one and, secondly, the mean value of the envelope defined by the local maxima and the envelope defined by the local minima must be zero. Thus, for a given signal $x(k)$ the appropriate EMD algorithm can be achieved according to these criteria. The upper envelope is created by cubic spline interpolation of the local maxima and the lower envelope is created by interpolation of the local minima. The first component can be obtained by subtracting the mean $m_{1}(k)$ calculated with the upper and lower envelopes from the original series $x(k)$.

$$
c_{1}(k)=x(k)-m_{1}(k)
$$

The resulting component $c_{1}(k)$ is the first IMF if the mode fulfils the definition of an IMF. If $c_{1}(k)$ is not an IMF, the sifting process has to be repeated as many times as is required to reduce the extracted signal to an IMF. Once this condition is reached, the first component $c_{1}(k)$ is separated from the original series $x(k)$ to leave the first residue $r_{1}(k)$ :

$$
r_{1}(k)=x(k)-c_{1}(k)
$$

The residue $r_{1}(k)$ now contains information about the components for a longer period, and therefore it is treated as the new data and is resifted to obtain additional components. The procedure will be continued until finally it meets a stopping criterion. By this procedure, lower order IMFs capture fast oscillation modes while higher order IMFs typically disclose slow oscillation modes. The original signal $x(k)$ can thus be expressed as follows:

$$
x(k)=\sum_{j=1}^{N} c_{j}(k)+r_{N}(k)
$$


where $N$ is the number of IMFs, and IMFs $c_{j}(k)(j=1, \ldots, N)$ are nearly orthogonal to each other and all have zero means, $r_{N}(k)$ is the final residue. The result of the EMD produces $N$ fundamental components and a residue signal. The residue itself is a very low frequency component or a trend, and can also be regarded as the last IMF.

\subsection{Multidistance Measurement and EMD-RLS Algorithm to Remove Global Interference}

A five-layer model of the adult human head, consisting of scalp, skull, cerebrospinal fluid (CSF), gray matter, and white matter was used here (see Figure 1). The use of particular source-detector separation allows us to distinguish between processes occurring at different tissue depths, as indicated by the "banana-shaped" regions that encompass statistically determined photon paths [21]. The true haemodynamic changes related to the specific stimuli present in the gray matter layer. Thus we use short interoptode distance with S-D1 to probe the superficial tissue layer, and long interoptode distance with SD2 to probe the deep tissue layer. In this approach, the near detector is positioned at $5 \mathrm{~mm}$ to $15 \mathrm{~mm}$ from the source and the far detector is positioned at more than $30 \mathrm{~mm}$ from the source [22]. In our study here, the short interoptode distance is chosen at $10 \mathrm{~mm}$ and the far interoptode distance is chosen at $40 \mathrm{~mm}$. To remove global interference in fNIRS measurement, short-distance measurements were therefore used as the reference channel to estimate global interference present with the long-distance measurement according to the following analysis.

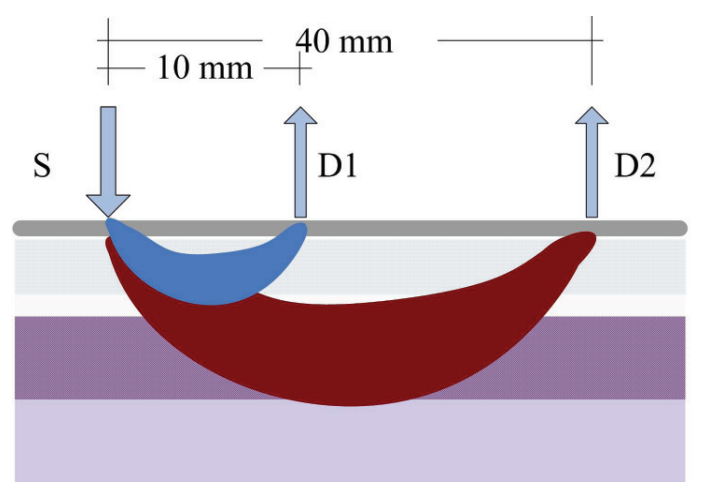

scalp

skull

$\mathrm{CSF}$

gray matter

white matter

FIG. 1 Schematic illustration of five-layered slab human head model and multidistance optode configuration. $\mathrm{S}$ is the photon source, and D1 and D2 are detectors.

The concentration changes of $\mathrm{HbO}_{2}$ and $\mathrm{HHb}$ in tissues can be obtained with a two wavelength $\left(\lambda_{1}\right.$ and $\left.\lambda_{2}\right)$ source. For a fixed source-detector distance, the change of optical density, $\Delta A$, and the concentration changes of $\mathrm{HbO}_{2}$ and $\mathrm{HHb}$ $\left(\Delta\left[\mathrm{HbO}_{2}\right]\right.$ and $\left.\Delta[\mathrm{HHb}]\right)$ can be represented by the modified Lambert-Beer law (MLBL).

$$
\left[\begin{array}{l}
\Delta A\left(\lambda_{1}\right) \\
\Delta A\left(\lambda_{2}\right)
\end{array}\right]=L\left[\begin{array}{ll}
\varepsilon_{\mathrm{HbO}_{2}}\left(\lambda_{1}\right) & \varepsilon_{\mathrm{HHb}}\left(\lambda_{1}\right) \\
\varepsilon_{\mathrm{HbO}_{2}}\left(\lambda_{2}\right) & \varepsilon_{\mathrm{HHb}}\left(\lambda_{2}\right)
\end{array}\right]\left[\begin{array}{c}
\Delta\left[\mathrm{HbO}_{2}\right] \\
\Delta[\mathrm{HHb}]
\end{array}\right]
$$

where $\varepsilon_{\mathrm{HbO}_{2}}(\lambda)$ and $\varepsilon_{\mathrm{HHb}}(\lambda)$ are the molar extinction coefficients of $\mathrm{HbO}_{2}$ and $\mathrm{HHb}$, respectively. Hereinto, $L$ is denoted as:

$$
L=\operatorname{diag}\left[D P F\left(\lambda_{1}\right) r, D P F\left(\lambda_{2}\right) r\right]
$$

where $\operatorname{DPF}(\lambda)$ is the differential path length factor at a wavelength $\lambda$, and $r$ is the linear distance from the source to the detector. The concentration changes of oxyhaemoglobin, $\Delta\left[\mathrm{HbO}_{2}\right]$, and deoxyhaemoglobin, $\Delta[\mathrm{HHb}]$, can be derived from Equation (4):

$$
\left[\begin{array}{c}
\Delta\left[\mathrm{HbO}_{2}\right] \\
\Delta[\mathrm{HHb}]
\end{array}\right]=\left[\begin{array}{ll}
\varepsilon_{\mathrm{HbO}_{2}}\left(\lambda_{1}\right) & \varepsilon_{\mathrm{HHb}}\left(\lambda_{1}\right) \\
\varepsilon_{\mathrm{HbO}_{2}}\left(\lambda_{2}\right) & \varepsilon_{\mathrm{HHb}}\left(\lambda_{2}\right)
\end{array}\right]^{-1} L^{-1}\left[\begin{array}{l}
\Delta A\left(\lambda_{1}\right) \\
\Delta A\left(\lambda_{2}\right)
\end{array}\right]
$$

where $L^{-1}$ denotes the inverse matrix of $L$. When we have measured the change of optical density, the time series of $\Delta\left[\mathrm{HbO}_{2}\right]$ and $\Delta[\mathrm{HHb}]$ obtained with S-D1 and S-D2 can be calculated using Equation (6).

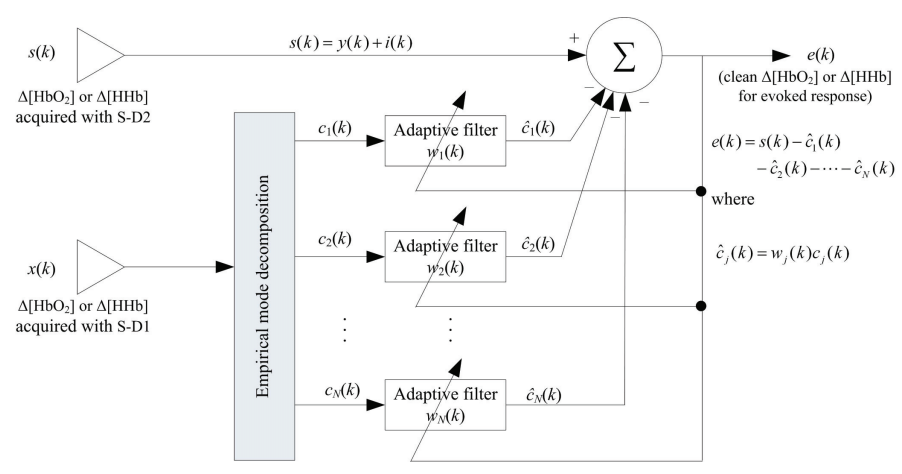

FIG. 2 Block diagram of global interference denoising using EMD-RLS algorithm in multidistance fNIRS measurement.

Figure 2 shows the block diagram of the reduction of global interference for fNIRS brain activity measurement in our study. The objective is to subtract the estimated global interference from the long-distance measurement. EMD was used here to decompose short-distance measurement into several IMFs according to the frequency characteristic.

$$
x(k)=\sum_{i=1}^{N} c_{i}(k)
$$

where $x(k)$ is the $\Delta\left[\mathrm{HbO}_{2}\right]$ or $\Delta[\mathrm{HHb}]$ acquired from the shortdistance measurement at instant $k, N$ is the number of IMF. Note that for convenience the residue term is absorbed in the summation as the last term. Then we consider the matter of mapping the IMFs of $x(k)$ in a fashion that approximates global interference $i(k)$, that is related to $x(k)$. The IMFs can be combined utilizing various possible methodologies under various objective functions designed to match $i(k)$. Mathematically, the linear fit is adopted here, as given by Equation (8):

$$
\hat{i}(k)=\sum_{i=1}^{N} w_{i}(k) c_{i}(k)
$$

where $\hat{i}(k)$ is the estimation of $i(k)$ and the coefficient $w_{i}(k)$ is the weighting assigned to the $i$ th IMF at instant $k$.

The haemodynamic changes acquired with S-D2 is modelled as a sum of the true brain activity response $y(k)$ and global interference $i(k)$. The true brain activity response can then be estimated as $e(k)$ :

$$
e(k)=s(k)-\hat{i}(k)=y(k)+[i(k)-\hat{i}(k)]
$$

where $s(k)$ is the $\Delta\left[\mathrm{HbO}_{2}\right]$ or $\Delta[\mathrm{HHb}]$ acquired from the longdistance measurement at instant $k$. To produce an estimation 
of global interference in the brain activity analysis, the following cost function of weighted least squares is chosen for the optimization criterion:

$$
J_{k}=\sum_{n=1}^{k} \chi^{k-n} e^{2}(k)
$$

The parameter $0<\chi \leq 1$ is the forgetting factor that controls the memory span of the algorithm. Using Equation (8) and Equation (9), the following equation can be obtained from Equation (10):

$$
J_{k}=\sum_{n=1}^{k} \chi^{k-n}\left[s(n)-\sum_{i=1}^{N} w_{i}(k) c_{i}(n)\right]^{2}
$$

Here, the weight $w_{i}(k)$ was used to adjust the proportion of each specific IMF in global interference. The optimal coefficient $w_{i}(k)$ for minimizing $J_{k}$ can be obtained by differentiating Equation (11) with respect to $w_{i}(k)$ and setting the derivatives to zero. This yields the following equation:

$$
\begin{aligned}
\frac{\partial J_{k}}{\partial w_{i}(k)}= & -2 \sum_{n=1}^{k}\left\{\chi^{k-n}[s(n)\right. \\
& \left.\left.-\sum_{i=1}^{N} w_{i}(k) c_{i}(n)\right] c_{j}(n)\right\}=0
\end{aligned}
$$

then we can obtain

$$
\sum_{n=1}^{k} \chi^{k-n} s(n) c_{j}(n)=\sum_{i=1}^{N} w_{i}(k) \sum_{n=1}^{k} \chi^{k-n} c_{i}(n) c_{j}(n)
$$

by defining

$$
\begin{aligned}
p_{j}(k) & =\sum_{n=1}^{k} \chi^{k-n} s(n) c_{j}(n) \\
R_{i j}(k) & =\sum_{n=1}^{k} \chi^{k-n} c_{i}(n) c_{j}(n)
\end{aligned}
$$

Then Equation (13) can be rewritten as

$$
\sum_{i=1}^{N} R_{i j}(k) w_{i}(k)=p_{j}(k), j=1,2, \ldots, N
$$

Considering all the values of the index $i$ and $j$, we can obtain the following equation with the matrix form.

$$
\left[\begin{array}{cccc}
R_{11}(k) & R_{12}(k) & \cdots & R_{1 N}(k) \\
R_{21}(k) & R_{22}(k) & \cdots & R_{2 N}(k) \\
\vdots & \vdots & \ddots & \vdots \\
R_{N 1}(k) & R_{N 2}(k) & \cdots & R_{N N}(k)
\end{array}\right]\left[\begin{array}{c}
w_{1}(k) \\
w_{2}(k) \\
\vdots \\
w_{N}(k)
\end{array}\right]=\left[\begin{array}{c}
p_{1}(k) \\
p_{2}(k) \\
\vdots \\
p_{N}(k)
\end{array}\right]
$$

This can be briefly denoted as

$$
\mathbf{R}(k) \mathbf{w}(k)=\mathbf{p}(k)
$$

The optimal coefficients can be acquired by the following equation.

$$
\mathbf{w}(k)=\mathbf{R}^{-1}(k) \mathbf{p}(k)
$$

Since the straightforward computation of the inverse of $\mathbf{R}^{-1}(k)$ results in an algorithm with computational complexity of $O\left[N^{3}\right]$, we implement the computation of the inverse matrix by means of the Matrix Inversion Lemma [23].

$$
\begin{aligned}
\mathbf{Q}(k)=\mathbf{R}^{-1}(k)= & \frac{1}{\chi}[\mathbf{Q}(k-1) \\
& \left.-\frac{\mathbf{Q}(k-1) \mathbf{c}(k) \mathbf{c}^{T}(k) \mathbf{Q}(k-1)}{\chi+\mathbf{c}^{T}(k) \mathbf{Q}(k-1) \mathbf{c}(k)}\right]
\end{aligned}
$$

where

$$
\mathbf{c}(k)=\left[c_{1}(k), c_{2}(k), \ldots, c_{N}(k)\right]^{T}
$$

We define the a prior error as

$$
e^{\prime}(k)=s(k)-\mathbf{c}(k) \mathbf{w}(k-1)
$$

By expressing $s(k)$ as a function of the a prior error [23] and the weight vector $\mathbf{w}(k)$ can be expressed as

$$
\mathbf{w}(k)=\mathbf{w}(k-1)-e^{\prime}(k) \mathbf{Q}(k) \mathbf{c}(k)
$$

From the above analysis, $e(k)$ is the estimated value of the true brain activity response $y(k)$. The critical aspect of using EMDRLS here is to optimize the estimation of global interference utilizing linear mapping between the decomposed IMFs and the long-distance measurement.

\section{Method}

In the present study we use one light source (S) and two light detectors (D1 and D2) shown in Figure 1. One light source with two wavelengths, 750 and $830 \mathrm{~nm}$, and two detectors were positioned on the surface of the medium to collect diffuse reflectance data. The light source and the detectors are all positioned perpendicular to the surface of the medium. A five-layer slab model consisting of scalp (sc), skull (sk), cerebrospinal fluid (CSF), gray matter (gm), and white matter (wm) was used to represent a human adult head.

\subsection{Monte Carlo Simulations}

To determine the effectiveness of our algorithm, Monte Carlo simulations of the five-layered human head model were performed. The simulations were carried out using the typical fNIRS probe arrangement illustrated in Figure 1.

The Monte Carlo code used here is an extension of the general multi-layer, three dimensional, weighted photon Monte Carlo codes developed by Wang et al [24]. Scattering anisotropies were assumed to be 0.9 and Fresnel reflection at the tissueair boundary was also considered. We assumed the same refractive index of $n=1.4$ for all layers [25]. The standard parameters used in the simulation are given in Table 1 . Thickness, transport scattering coefficient $\mu_{s}^{\prime}, \mathrm{HbO}_{2}$ and $\mathrm{HHb}$ baseline concentrations were taken from published data [26-28]. The baseline concentration of $\mathrm{HbO}_{2}$ and $\mathrm{HHb}$ assumed that the oxygen saturation in the head was $70 \%$. Absorption coefficients were calculated with the $\mathrm{HbO}_{2}$ and $\mathrm{HHb}$ baseline concentrations and the molar extinction coefficients. The molar extinction coefficients at 750 and $830 \mathrm{~nm}$ were obtained from the literature [29]. 


\begin{tabular}{cccccc}
\hline & \multicolumn{2}{c}{ Baseline Concentration } & $\begin{array}{c}\text { Absorption } \\
\text { coefficient } \mu_{a}\end{array}$ & $\begin{array}{c}\text { Transport scattering } \\
\text { coefficient } \mu_{s}^{\prime}\end{array}$ & $\begin{array}{c}\text { Thickness } \\
\text { Tissue type }\end{array}$ \\
\cline { 2 - 3 } & $\mathrm{HbO}_{2}(\mu \mathrm{M})$ & $\mathrm{HHb}(\mu \mathrm{M})$ & $750 / 830 \mathrm{~nm}\left(\mathrm{~mm}^{-1}\right)$ & $750 / 830 \mathrm{~nm}\left(\mathrm{~mm}^{-1}\right)$ & $(\mathrm{mm})$ \\
\hline Scalp & 64 & 27 & $0.0177 / 0.0203$ & $2.11 / 1.84$ & 3 \\
Skull & 57 & 24 & $0.0158 / 0.0181$ & $1.79 / 1.47$ & 7 \\
CSF & 14 & 6 & $0.0039 / 0.0045$ & $0.27 / 0.22$ & 2 \\
Gray matter & 128 & 55 & $0.0358 / 0.0409$ & $2.39 / 2.10$ & 4 \\
White matter & 50 & 21 & $0.0138 / 0.0158$ & $9.62 / 8.82$ & 30 \\
\hline
\end{tabular}

TABLE 1 Haemodynamic parameters, thickness, and optical properties for each layer of an adult head model.

\subsection{Generation of Time Series of Haemodynamic Changes and Physiological Interference}

The haemodynamic changes were simulated as a combination of the functional haemodynamic responses and the physiological interference. The functional haemodynamic responses in gray matter were defined as the convolution of the stimulation $s(t),[s(t)=0$ for rest period, and 1 for stimulation $]$ and a prototypical haemodynamic impulse $h(t)[15,26,30]$.

$$
\begin{aligned}
& s(t)= \begin{cases}0 & t \in \text { rest } \\
1 & t \in \text { simulation }\end{cases} \\
& h(t)=t^{b} \exp \left(-\frac{t}{d}\right)
\end{aligned}
$$

The parameters $b$ and $d$ in the gamma variate function were set at 8.6 and 0.56 respectively, which corresponds to recent findings $[26,30]$.

The evoked haemodynamic response $u(t)$ was the convolution of $s(t)$ and $h(t)$ :

$$
u(t)=K[h(t) * s(t)]
$$

where $K$ is a simple scaling factor in our model. In order to make the simulation as realistic as possible we generated physiological interference by a combination of cardiac fluctuation $c(t)$,respiratory fluctuations $r(t)$, low frequency oscillation $m(t)$, very low frequency oscillation $v(t)$, and independent fluctuation $\tau(t)$ induced by the temperature changes and the sweat on the skin. The haemodynamic changes in each layer are denoted as follows:

$$
\begin{aligned}
& C_{\mathrm{HbO}_{2}}^{1}(t)=\mathrm{HbO}_{2 \mathrm{base}}^{1}+\tau(t)\left[\alpha_{\mathrm{HbO}_{2}}^{1} c(t)+\beta_{\mathrm{HbO}_{2}}^{1} r(t)\right. \\
& \left.+\gamma_{\mathrm{HbO}_{2}}^{1} m(t)+\zeta_{\mathrm{HbO}_{2}}^{1} v(t)+\iota_{\mathrm{HbO}_{2}}^{1} u(t)\right] \\
& C_{\mathrm{HHb}}^{1}(t)=\mathrm{HHb}_{\text {base }}^{1}+\tau(t)\left[\alpha_{\mathrm{HHb}}^{1} c(t)+\beta_{\mathrm{HHb}}^{1} r(t)\right. \\
& \left.+\gamma_{\mathrm{HHb}}^{1} m(t)+\zeta_{\mathrm{HHb}}^{1} v(t)+\iota_{\mathrm{HHb}}^{1} u(t)\right] \\
& C_{\mathrm{HbO}_{2}}^{2,3,4,5}(t)=\mathrm{HbO}_{2 \mathrm{base}^{2,4,5}}^{2,4,}+\alpha_{\mathrm{HbO}_{2}}^{2,3,4,5} c(t)+\beta_{\mathrm{HbO}_{2}}^{2,3,4,5} r(t) \\
& +\gamma_{\mathrm{HbO}_{2}}^{2,3,4,5} m(t)+\zeta_{\mathrm{HbO}_{2}}^{2,3,4,5} v(t)+\iota_{\mathrm{HbO}_{2}}^{2,3,4,5} u(t) \\
& C_{\mathrm{HHb}}^{2,3,4,5}(t)=\mathrm{HHb}_{\text {base }}^{2,3,4,5}+\alpha_{\mathrm{HHb}}^{2,3,4,5} c(t)+\beta_{\mathrm{HHb}}^{2,3,4,5} r(t) \\
& +\gamma_{\mathrm{HHb}}^{2,3,4,5} m(t)+\zeta_{\mathrm{HHb}}^{2,3,4,5} v(t)+\iota_{\mathrm{HHb}}^{2,3,4,5} u(t)
\end{aligned}
$$

where $C_{\mathrm{HbO}_{2}}^{1}(t), C_{\mathrm{HHb}}^{1}(t), C_{\mathrm{HbO}_{2}}^{2,3,4,5}(t)$, and $C_{\mathrm{HHb}}^{2,3,4,5}(t)$ represent the concentration of $\mathrm{HbO}_{2}$ and $\mathrm{HHb}$ in each layer as a function of time, with the superscripts 1 to 5 indicating the layer index for scalp, skull, CSF, gray and white matters, respectively. $\mathrm{HbO}_{2}$ base and $\mathrm{HHb}_{\text {base }}$ represent average or baseline concentrations. The cardiac fluctuation $c(t)$, respiratory fluctuation $r(t)$, low frequency oscillation $m(t)$, and the very low frequency oscillation $v(t)$ were all simulated as the summation of sinusoidal wave and white Gaussian noise. The form of $c(t), \quad r(t), \quad m(t)$, and $v(t)$ is defined as $\quad c(t)=\sin \left(2 \pi f_{c} t\right)+\sigma_{c}(t), r(t)=\sin \left(2 \pi f_{r} t\right)+\sigma_{r}(t)$, $m(t)=\sin \left(2 \pi f_{m} t\right)+\sigma_{m}(t), v(t)=\sin \left(2 \pi f_{v} t\right)+\sigma_{v}(t)$, respectively. The frequency values of each sine wave were defined according to the mean frequencies of real signals $\left(f_{c}=1 \mathrm{~Hz}\right.$, $\left.f_{r}=0.25 \mathrm{~Hz}, f_{m}=0.1 \mathrm{~Hz}, f_{v}=0.04 \mathrm{~Hz}\right)[5,31-33]$. The coefficients $\alpha, \beta, \gamma, \zeta$ and $\iota$ with layer index as superscript and $\mathrm{HHb}$ or $\mathrm{HbO}_{2}$ as subscript are the haemodynamic variation amplitude control parameters, which can be found in Table 2. The independent interference $\tau(t)$ is generated by biased and low pass filtered Gaussian white noise. The independent interference $\tau(t)$ in the scalp layer and the different weight on cardiac, respiratory, and low-frequency and very low frequency fluctuations in each layer were to simulate a certain amount of uncorrelated changes in the superficial layers compared with deep layers. The parameters used are based on Scholkmann et al. [33] and Zhang et al [15].

The simulated haemodynamic changes were used to calculate the optical measurement by Monte Carlo method. The sampling rate was set to $10 \mathrm{~Hz}$ and the whole time series for the changes of optical density were acquired under the assumption that the scattering properties of the head do not vary with time. The experiment is designed as a 5-epoch block and each individual epoch consisted of a series of 400 points, 200 points of rest and 200 points of stimulation.

\section{Results}

\subsection{Intrinsic Mode Functions of Haemodynamic Changes with Short Interoptode Distance}

Partial pathlength (PPL) is the mean path length of the photons travelling in a specified region, which could reflect the effective transmitting path for the photons in each layer. According to the MLBL, the partial pathlength is the key factor that determines the light attenuation under the assumption of constant absorption and scattering coefficients. In our simula- 


\begin{tabular}{|c|c|c|c|c|c|c|}
\hline Head layers & $\begin{array}{c}\text { Blood } \\
\text { contents }\end{array}$ & $\begin{array}{c}\alpha(\mu \mathrm{M}) \\
\text { respiration }\end{array}$ & $\begin{array}{l}\beta(\mu \mathrm{M}) \\
\text { cardiac }\end{array}$ & $\begin{array}{c}\gamma(\mu \mathrm{M}) \\
\text { low frequency } \\
\text { oscillation }\end{array}$ & $\begin{array}{c}\zeta(\mu \mathrm{M}) \\
\text { very low frequency } \\
\text { oscillation }\end{array}$ & $\begin{array}{c}\iota(\mu \mathrm{M}) \\
\text { evoked } \\
\text { response }\end{array}$ \\
\hline \multirow{2}{*}{ Scalp } & $\mathrm{HbO}_{2}$ & 0.2 & 0.6 & 0.9 & 1.0 & 0 \\
\hline & $\mathrm{HHb}$ & 0.013 & 0.04 & 0.058 & 0.06 & 0 \\
\hline \multirow{2}{*}{ Skull } & $\mathrm{HbO}_{2}$ & 0.2 & 0.63 & 0.96 & 1.1 & 0 \\
\hline & $\mathrm{HHb}$ & 0.012 & 0.045 & 0.06 & 0.07 & 0 \\
\hline \multirow{2}{*}{ CSF } & $\mathrm{HbO}_{2}$ & 0.02 & 0.06 & 0.08 & 0.11 & 0 \\
\hline & $\mathrm{HHb}$ & 0.001 & 0.004 & 0.005 & 0.007 & 0 \\
\hline \multirow{2}{*}{ Gray matter } & $\mathrm{HbO}_{2}$ & 0.2 & 0.65 & 0.92 & 1.1 & 15 \\
\hline & $\mathrm{HHb}$ & 0.014 & 0.043 & 0.07 & 0.072 & -4 \\
\hline \multirow{2}{*}{ White matter } & $\mathrm{HbO}_{2}$ & 0.2 & 0.6 & 0.9 & 0.9 & 0 \\
\hline & $\mathrm{HHb}$ & 0.012 & 0.04 & 0.06 & 0.06 & 0 \\
\hline
\end{tabular}

TABLE 2 Simulation parameters for amplitude and frequency of interference oscillations and haemodynamic changes.

tions for the short interoptode distance, the partial pathlength in the gray matter $\left(\mathrm{PPL}_{\mathrm{gm}}\right)$ is very small, the value of $\mathrm{PPL}_{\mathrm{gm}}$ being about $10^{-2.6} \times$ that in the superficial layer. Thus, the optical measurement made at a short interoptode distance could be very appropriate to be used to estimate global interference, where the superficial variation or non-activation signals dominate the measurement.

The simulated optical measurements at 750 and $830 \mathrm{~nm}$ were acquired with Monte Carlo simulations. The changes of optical density for the $10 \mathrm{~mm}$ source-detector distance are shown in Figure 3. The shaded regions in the figure indicate the periods of evoked stimulation. The changes of optical density should be zero under ideal conditions if no interference is present. As shown in Figure 3, the fluctuations are evident in the changes of optical density for both 750 and $830 \mathrm{~nm}$.

The concentration changes of $\mathrm{HbO}_{2}$ and $\mathrm{HHb}\left(\Delta\left[\mathrm{HbO}_{2}\right]\right.$ and $\Delta[\mathrm{HHb}])$ were calculated with the MLBL, which has been described in Equation (6). The calculated $\Delta\left[\mathrm{HbO}_{2}\right]$ and $\Delta[\mathrm{HHb}]$ contain several components of interference, including those induced by events related to the cardiac cycle and to breathing, as well as by spontaneous physiological low-frequency oscillations, and very low frequency oscillations. The EMD method was utilized to produce the IMF components for $\Delta\left[\mathrm{HbO}_{2}\right]$ and $\Delta[\mathrm{HHb}]$. As shown in Figure 4 and Figure 5, five IMF components and a residue were generated with EMD for $\Delta\left[\mathrm{HbO}_{2}\right]$, and five IMF components and a residue were generated with EMD for $\Delta[\mathrm{HHb}]$.

It is clearly demonstrated in Figure 4 and Figure 5 that EMD can successfully decompose the signals of interest into components whose frequency relationship is fully consistent with the known sources of interference. The cardiac pulse is clearly evident in the first IMF, breathing is found in the second IMF, the low frequency oscillation is found in the third IMF, and the very low frequency oscillation is found in the fourth IMF. The additional IMF components presented in the decomposition process are mainly due to the introduction of a slowly varying random signal as the independent interference, which was simulated as the uncorrected superficial response compared with the intracerebral response.

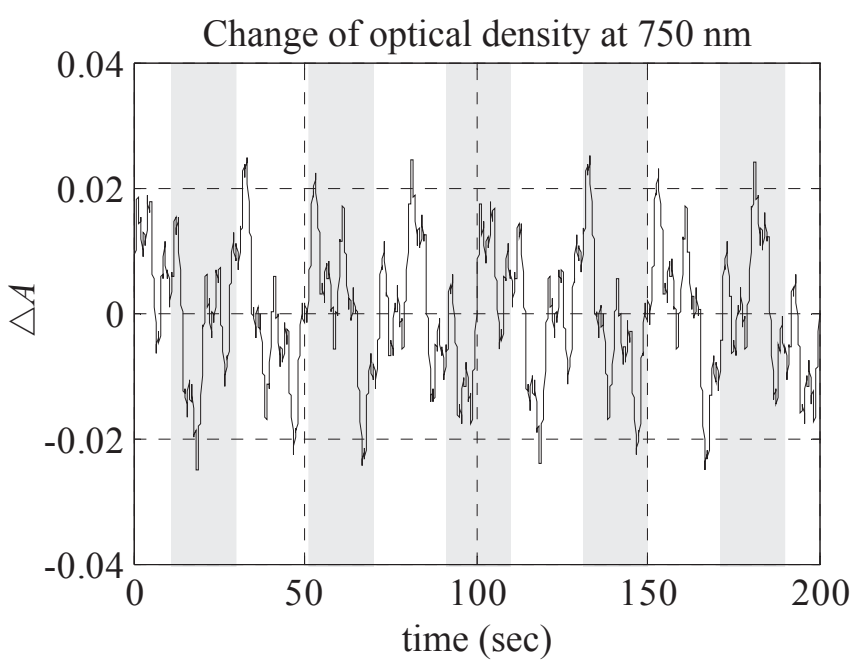

(a)

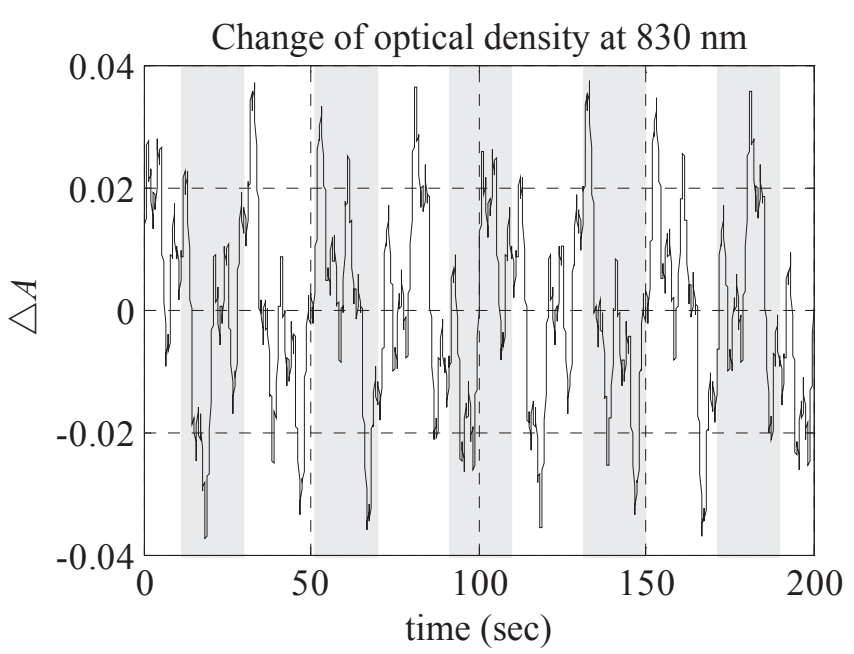

(b)

FIG. 3 Simulated measurements of optical density for the $10 \mathrm{~mm}$ source-detector distance: (a) change of optical density for $750 \mathrm{~nm}$, and (b) change of optical density for $830 \mathrm{~nm}$.

\subsection{Application of EMD-RLS for Global Interference Reduction}

After the IMFs are acquired, the data analysis described in Section 2.2 is applied. From the results as shown in Figure 4 


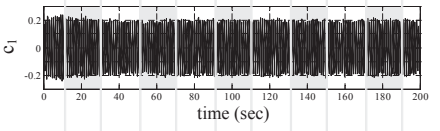

(a)

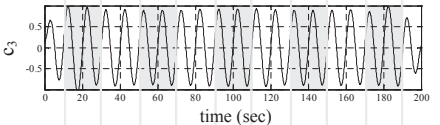

(c)

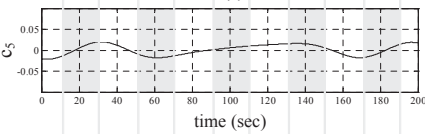

(e) (b)
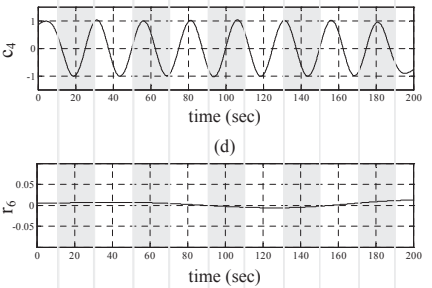

(f)

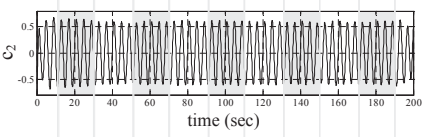

FIC. 4 The IMF components of concentration changes of $\mathrm{HbO}_{2}$ acquired with shortdistance optode. The units of the $y$-axis for IMFs are micromole $\left({ }^{-} \mathrm{M}\right)$.

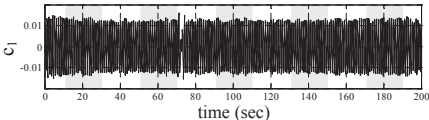

(a)

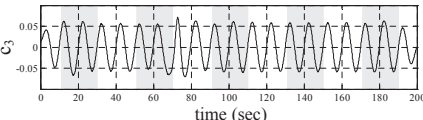

(c)

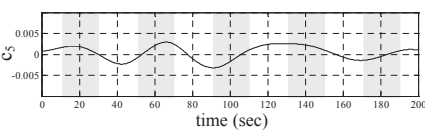

(e)

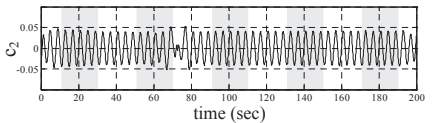

(b)

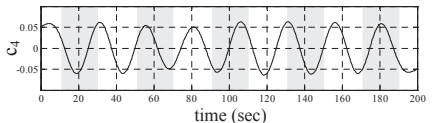

(d)

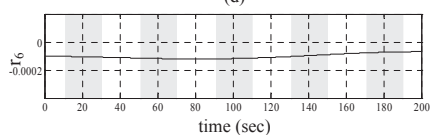

(f)
FIC. 5 The IMF components of concentration changes of $\mathrm{HHb}$ acquired with shortdistance optode. The units of the $y$-axis for IMFs are micromole $\left({ }^{-} \mathrm{M}\right)$

and Figure 5, the number of IMFs $N=6$. The forgetting factor $\chi$ in our study was set to 0.99999 , which is relatively stable in our tests. The total signal length is 3000 samples and the first 1000 samples are used to train the signal and obtain the weighting coefficients assigned to the specified component (see Table 3). After the optimal coefficients are determined, the IMFs are applied to approximate global interference. The remaining 2000 samples are then applied for interference cancellation by subtracting the estimated global interference. As seen in Table 3, the coefficients for each component are different. It can be seen that the magnitudes of the first four coefficients are larger than those of the last two coefficients. This can be interpreted that the first four IMFs are the interference components corresponding to heartbeat, breathing, low frequency oscillation and very low frequency oscillation, which are correlated with global interference.

Concentration changes of $\mathrm{HbO}_{2}$ during the simulated measurement are shown in Figure 6. Thereinto, Figure 6a shows the $\Delta\left[\mathrm{HbO}_{2}\right]$ time series results from a $10 \mathrm{~mm}$ source-detector distance that were calculated with the MLBL and Figure $6 \mathrm{~b}$ shows the block average result. Similarly, Figure $6 \mathrm{c}$ and $6 \mathrm{~d}$ show the $\Delta\left[\mathrm{HbO}_{2}\right]$ time series and block average results from a $40 \mathrm{~mm}$ source-detector distance, again calculated with the MLBL. However, neither the results from Figure $6 \mathrm{c}$ nor $6 \mathrm{~d}$ can clearly reveal the true activation signal, since it is obvious that the raw time series has been contaminated by global interference. After signal processing with the EMD-RLS, as seen in Figure $6 e$ and $6 f$, the evoked haemodynamic changes are clear
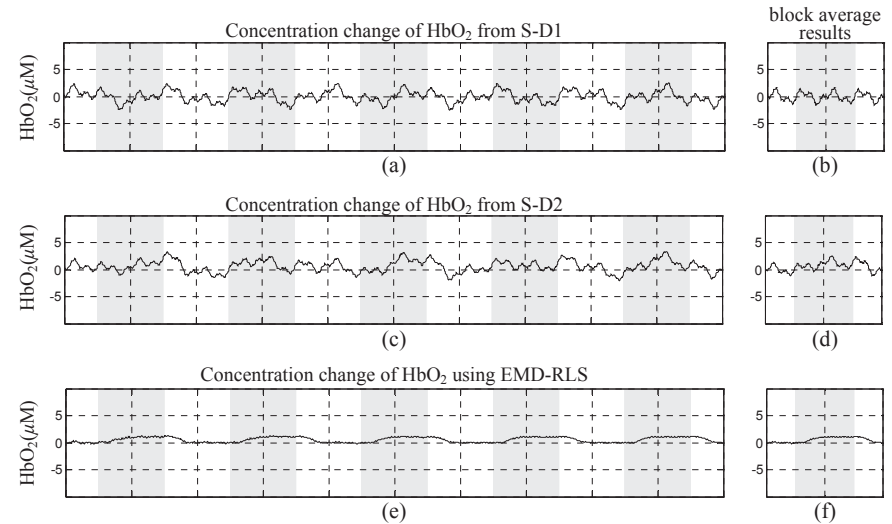

Concentration change of $\mathrm{HbO}_{2}$ using EMD-RLS with PVE compensation

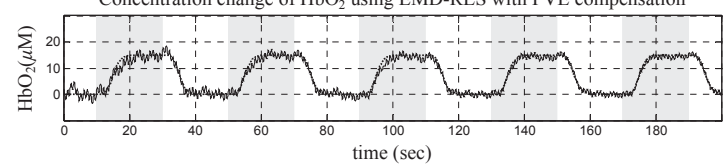

(g)

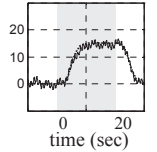

(h)

FIG. 6 EMD-RLS to remove global interference. (a) The time series of concentration changes of $\mathrm{HbO}_{2}$ calculated from S-D1 with $10 \mathrm{~mm}$ source-detector separation, and (b) its block averaged result. (c) and (d) The time series of concentration changes of $\mathrm{HbO}_{2}$ calculated from S-D2 with $40 \mathrm{~mm}$ source-detector separation. (e) and (f) EMD-RLS result for the target measurement. (g) and (h) EMD-RLS result with PVE compensation (solid line), together with the true evoked brain activity (dashed line) used for quantitative comparison and evaluation of the performance of EMD-RLS algorithm.

and most of global interference has been removed. However, the magnitude of $\Delta\left[\mathrm{HbO}_{2}\right]$ is underestimated when calculating with the MLBL because the partial path length in the gray matter is always smaller than the differential path length. This is generally referred to as the partial volume effect (PVE) [34]. By means of Monte Carlo simulations, the ratio of the differential path length to the partial path length in the gray matter can be achieved to compensate for the PVE effect. After we have compensated for the PVE effect in the recovered results we can compare the recovered results with the real evoked haemodynamic changes in the gray matter. This comparison can be seen in Figure $6 \mathrm{~g}$ and $6 \mathrm{~h}$. In these two figures, the solid line denotes the recovered results with PVE compensation and the dashed line denotes the true evoked haemodynamic changes used in the simulation. Although some fluctuations still remain, the recovered $\Delta\left[\mathrm{HbO}_{2}\right]$ processed with the EMD-RLS algorithm provide an obvious evoked response. By calculating ensemble average for the whole time series, the results demonstrate that the proposed methodology could remove approximately $90 \%$ of global interference.

Figure 7 shows the equivalent results of Figure 6 but for $\Delta[\mathrm{HHb}]$. Comparing Figure $6 \mathrm{a}$ and $6 \mathrm{~b}$ with Figure $7 \mathrm{a}$ and $7 \mathrm{~b}$, respectively, we can observe that the interference in the superficial layer that was introduced into the $\Delta[\mathrm{HHb}]$ is relatively smaller than that in $\Delta\left[\mathrm{HbO}_{2}\right]$. Thus, the trend of $\Delta[\mathrm{HHb}]$ shown in Figure 7c and 7d was visible before EMD-RLS processing. The recovered results for $\Delta[\mathrm{HHb}]$ and its block average results are shown in Figure 7e and 7f. Although the interference in $\Delta[\mathrm{HHb}]$ is very small, the calculations shown in Figure $7 \mathrm{e}$ and $7 \mathrm{f}$ demonstrated that processed results are still somewhat better than the unprocessed results. The quantitative comparison [Figure $7 \mathrm{~g}$ and $7 \mathrm{~h}$ ] was conducted with the PVE compensation and approximately $91 \%$ of the $\Delta[\mathrm{HHb}]$ 


\begin{tabular}{ccccccc}
\hline IMF order $i$ & 1 & 2 & 3 & 4 & 5 & 6 \\
\hline$w_{i}$ for $\mathrm{HbO}_{2}$ & 0.5437 & 0.8034 & 0.8130 & 0.8384 & 0.0541 & 0.0685 \\
$w_{i}$ for $\mathrm{HHb}$ & 0.1756 & 0.7280 & 0.8723 & 1.1101 & 0.0394 & 0.0511 \\
\hline
\end{tabular}

TABLE 3 Optimal weighting coefficients assigned to the interference components.

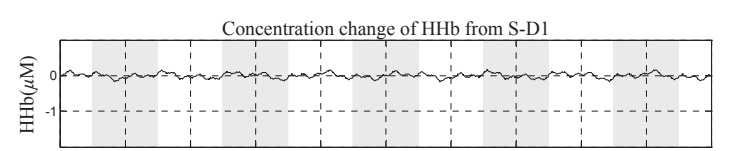

(a)

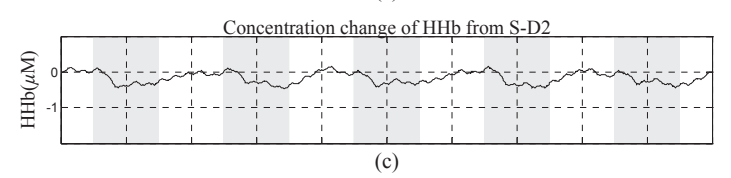

(c)
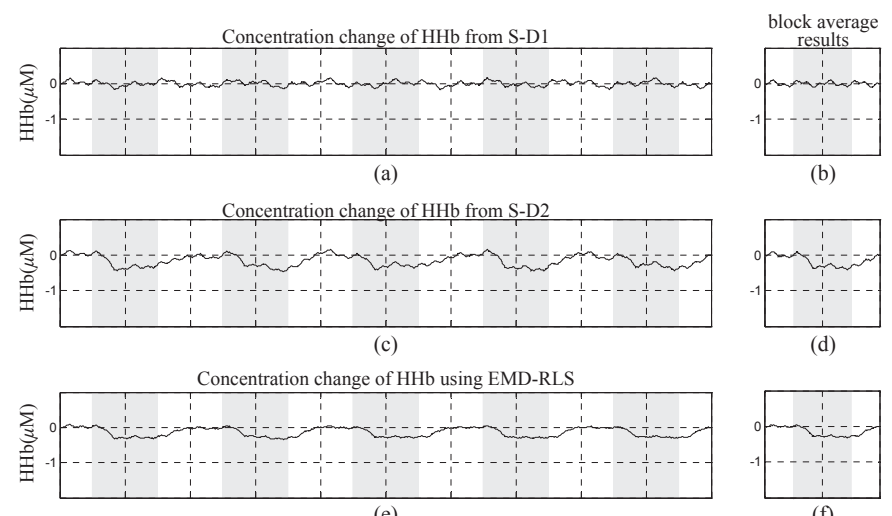

(b)

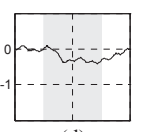

(d)

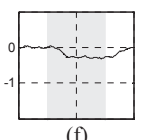

(f)

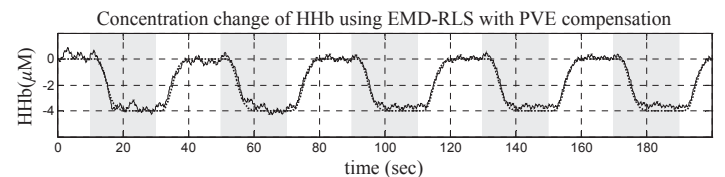

(g)

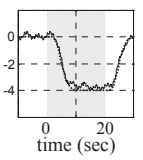

(h)

FIC. 7 EMD-RLS to remove global interference. (a) The time series of concentration changes of HHb calculated from S-D1 with $10 \mathrm{~mm}$ source-detector separation, and (b) its block averaged result. (c) and (d) The time series of concentration changes of $\mathrm{HHb}$ calculated from S-D2 with $40 \mathrm{~mm}$ source-detector separation. (e) and (f) EMD-RLS result for the target measurement. (g) and (h) EMD-RLS result with PVE compensation (solid line), together with the true evoked brain activity (dashed line) used for quantitative comparison and evaluation of the performance of EMD-RLS algorithm.

was recovered. We have also run the simulations for $5 \mathrm{~mm}$ and $45 \mathrm{~mm}$ short and long spacing respectively and the results are just as clear.

\section{Discussion}

Global interference can greatly degrade the performance of fNIRS measurement of evoked brain activity response. An approach to help identify and separate the interference components in fNIRS is to include auxiliary physiological measurements and to analyze the signal with an adaptive filtering algorithm [14,17]. Many instruments such as the pulse oximeter, electrocardiogram (ECG), chest band respirometer, spirometer and capnograph can be used to achieve auxiliary physiological measurements. This method is effective in reducing global interference, but the indispensability of additional equipments is the limitation of its application. Prince et al. [16] and Adbelnour et al. [18] utilized sine/cosine terms and Kalman filtering to model the specific physiological noise. However, this method needs to assume a prior frequencies (e.g. the cardiac, $\sim 1 \mathrm{~Hz}$; respiratory, $\sim 0.25 \mathrm{~Hz}$; and Mayer wave frequency, $\sim 0.1 \mathrm{~Hz}$ ) for the sine/cosine terms. In our previous work, the methodology based on EMD-HSA has also been presented good performance in removing cardiac and respiratory interference [19]. However, this methodology is not appropriate for the interference induced by low fre- quency oscillations, very low frequency oscillations, etc. Thus, a novel method based on EMD-RLS algorithm and multidistance probe configuration was proposed in this paper.

In this paper, EMD algorithm adaptively decomposes the superficial haemodynamic changes into several interference components and RLS algorithm dynamically adjusts the weighting of the corresponding interference to estimate global interference. Although EMD has been widely used to decompose complex signals and the RLS method has been broadly adopted for denoising in many domains, the approach of combination of EMD and RLS has not been applied for denoising in fNIRS brain activity measurement.

We have used Monte Carlo simulations of a five-layered model of the human adult head to assess the effectiveness of EMD-RLS in global interference removal from fNIRS brain activity data. Our results have shown that the method can reduce global interference within measurements of $\Delta\left[\mathrm{HbO}_{2}\right]$ and $\Delta[\mathrm{HHb}$. It should be noted that the EMD-RLS method is more effective for global interference removal in $\Delta\left[\mathrm{HbO}_{2}\right]$ than in $\Delta[\mathrm{HHb}]$ in our study. The reason could be interpreted that the interference magnitude of $\Delta\left[\mathrm{HbO}_{2}\right]$ is very large and the improvement of denoising for $\Delta\left[\mathrm{HbO}_{2}\right]$ is obvious.

An important matter that should be considered here is the traditional EMD algorithm adopts the cubic spline interpolation as an effective tool processing non-stationary signal and several issues may occur during the decomposition process. One of the issues is the presence of overshoot and undershoot found during the decomposition of the second IMF in $\Delta[\mathrm{HHb}]$. In order to solve the problem, many researchers attempted to overcome the overshoot [35], for example Qin et al. [36] used a Segment Slide Theory and Fan et al. [37] presented the piecewise linear fractal interpolation as the spline interpolating. Another issue is the modal distortion and mixture $[38,39]$ that often influence the performance of the EMD. Although this issue is not observed in our simulation study, the resolution of this issue may be useful in our future study for the EMD-RLS algorithm. The effective EMD methods based on independent component analysis [38] and the masking signal [39] were proposed to overcome this problem. Thus, some deeper investigations of EMD should be able to improve the performance of the EMD-RLS further.

One of the additional issues in fNIRS is that there can be a further source of interference, which is observed synchronously with a heart rate increase during the specific task (e.g. finger opposition task) [40,41]. This kind of interference is not independent of the functional response itself and thus using short-distance measurement as the reference channel may over-correct the target measurements. For these cases, methods considering the spatial locality difference between the brain functional response and global interference have been 
proposed based on principle component analysis [11,41] or independent component analysis [10]. The EMD-RLS algorithm could still be applicable for these cases if we put the target channel on the activated area and the reference channel on the unactivated area.

A truly rigorous evaluation of this method in vivo requires an uncontaminated evoked brain activity response signal, which, unfortunately, is unavailable. Meanwhile, the PVE effect in vivo can not be exactly compensated and the quantitative comparison of the recovery response and the true response of brain activity is difficult. Thus, Monte Carlo simulation is implemented for quantitative analysis here as the preliminary study. As for the test of this method in vivo with practical NIRS measurement, other signal processing methods such as power spectrum density will be utilized as auxiliary evaluation tool. This is the subject of our on-going research and will be published in due course.

\section{Conclusions}

The empirical mode decomposition is a promising tool for analyzing fNIRS signals through the frequency characteristic of specified IMFs. However, the optimality for the reconstruction of the given signal for IMFs is not considered. Thus, EMD-RLS algorithm based on multidistance probe configuration is used to study global interference reduction in fNIRS brain activity measurement. The evaluation of this method is carried out through simulating the evoked haemodynamic response and physiological interference. The method is effective to remove global interference induced not only by heartbeat and respiration but also by low frequency oscillation and very low frequency oscillation, and other correlated interference between superficial and deep layer. The advantage of EMD-RLS in multidistance fNIRS measurement compared with other possible methods also arises from its convenient implementation; it neither requires an auxiliary measurement instrument nor the dependence on a prior knowledge of the global interference frequency. Thus, the methodology has clear potential for use in fNIRS brain activity measurement.

\section{Acknowledgement}

The authors are grateful for the support from the Scitech Innovation Manpower Foundation of Harbin (No. 2009RFLXS006), the Sci-tech Innovation Foundation of Harbin Institute of Technology (assisted in 2008), and OBHL, UK. We also thank colleagues in China, Italy, UK and Japan for their helpful comments on this work.

\section{References}

[1] F. F. Jöbsis, "Noninvasive infrared monitoring of cerebral and myocardial oxygen sufficiency and circulatory parameters" Science 198, 1264-1267 (1977).

[2] B. Montcel, R. Chabrier, and P Poulet, "Detection of cortical activation with time-resolved diffuse optical methods" Appl. Optics 44, 1942-1947 (2005).
[3] S. Schmidt, C. M. Schwärzler, F. Sierra, M. Meyer-Wittkopf, and P. Rolfe, "Blood volume changes and oxygenation during labora laser spectroscopic analysis" Z. Geburtsh. Neonatol. 205, 33-37 (2001).

[4] P. A. Rea, J. Crowe, Y. Wickramasinghe, and P. Rolfe, “Non-invasive optical methods for the study of cerebral metabolism in the human newborn: a technique for the future?" J. Med. Eng. Technol. 9. 160-166 (1985).

[5] H. Obrig, M. Neufang, R. Wenzel, M. Kohl, J. Steinbrink, K. Einhäupl, and A. Villringer, "Spontaneous low frequency oscillations of cerebral hemodynamics and metabolism in human adults" Neurolmage $12,623-639$ (2000).

[6] L. F. Leonardo, D. M. Hueber, and T. J. Barstow, "Effects of assuming constant optical scattering on measurements of muscle oxygenation by near-infrared spectroscopy during exercise" J. Appl. Physiol. 102, 358-367 (2007).

[7] G. Strangman, D. A. Bois, and J. P. Sutton, “Non-invasive neuroimaging using near-infrared light" Biol. Psychiat. 52, 679-693 (2002).

[8] A. P. Gibson, J. C. Hebden, and S. R. Arridge, "Recent advances in diffuse optical imaging" Phys. Med. Biol. 50, R1-R43 (2005).

[9] T. S. Leung, C. E. Elwell, and D. T. Delpy, "Estimation of cerebral oxy- and deoxy-haemoglobin concentration changes in a layered adult head model using near-infrared spectroscopy and multivariate statistical analysis" Phys. Med. Biol. 50, 5783-5798 (2005).

[10] S. Kohno, I. Miyai, A. Seiyama, I. Oda, A. Ishikawa, S. Tsuneishi, T. Amita, and K. Shimizu, "Removal of the skin blood flow artifact in functional near-infrared spectroscopic imaging data through independent component analysis" J. Biomed. Opt. 12, 062111 (2007).

[11] Y. H. Zhang, D. H. Brooks, M. A. Franceschini, and D. A. Boas, "Eigenvector-based spatial filtering for reduction of physiological interference in diffuse optical imaging" J. Biomed. 0pt. 10, 011014 (2005).

[12] G. Jasdzewski, G. Strangman, J. Wagner, K. K. Kwong, R. A. Poldrack, and D. A. Boas, "Differences in the hemodynamic response to event-related motor and visual paradigms as measured by near infrared spectroscopy" Neurolmage 20, 479-488 (2003).

[13] R. B. Saager and A. J. Berger, "Direct characterization and removal of interfering absorption trends in two-layer turbid media" J. Opt. Soc. Am. A 22, 1874-1882 (2005).

[14] G. Morren, M. Wolf, P. Lemmerling, U. Wolf, J. H. Choi, E. Gratton, L. De Lathauwer, and S. Van Huffel, "Detection of fast neuronal signals in the motor cortex from functional near infrared spectroscopy measurements using independent component analysis" Med. Biol. Eng. Comput. 42, 92-99 (2004).

[15] Q. Zhang, E. N. Brown, and G. E. Strangman, “Adaptive filtering for global interference cancellation and real-time recovery of evoked brain activity: a Monte Carlo simulation study" J. Biomed. 0pt. 12, 044014 (2007).

[16] S. Prince, V. Kolehmainen, J. P. Kaipio, M. A. Franceschini, D. Boas, and S. R. Arridge, "Time-series estimation of biological factors in optical diffusion tomography" Phys. Med. Biol. 48, 1491-1504 (2003).

[17] S. G. Diamond, T. J. Huppert, V. Kolehmainen, M. A. Franceschini, J. P. Kaipio, S. R. Arridge, and D. A. Boas, "Physiological system identification with the Kalman filter in diffuse optical tomography" Med. Image Comput. Comput. Assist Interv. 8, 649-656 (2005).

[18] A. F. Abdelnour and T. Huppert, "Real-time imaging of human brain function by near-infrared spectroscopy using an adaptive general linear model" Neurolmage 46, 133-143 (2009). 
[19] Y. Zhang, J. W. Sun, and P. Rolfe, "Monte Carlo study for physiological interference reduction in near-infrared spectroscopy based on Empirical Mode Decomposition" J. Mod. Optic. 57, 2159-2169 (2010).

[20] N. E. Huang, Z. Shen, S. R. Long, M. C. Wu, H. H. Shih, Q. Zheng, N. C. Yen, C. C. Tung, and H. H. Liu, "The empirical mode decomposition and the Hilbert spectrum for nonlinear and non-stationary time series analysis" Proc. R. Soc. Lond. Ser-A 454, 903-995 (1998).

[21] P. Rolfe, "In vivo near-infrared spectroscopy" Annu. Rev. Biomed. Eng. 2, 715-754 (2000).

[22] R. Saager and A. Berger, "Measurement of layer-like hemodynamic trends in scalp and cortex: implementations for physiological baseline suppression in functional near-infrared spectroscopy" J. Biomed. Opt. 13, 034017 (2008).

[23] P. S. R. Diniz, Adaptive Filtering: Algorithms and Practical Implementation, Springer Science + Business Media, LLC, New York (2008).

[24] L. H. Wang, S. L. Jacques, and L. Q. Zheng, “MCML-Monte Carlo modeling of light transport in multi-layered tissues" Comput. Meth. Prog. Bio. 47, 131-146 (1995).

[25] E. Okada and D. T. Delpy, "Near-infrared light propagation in an adult head model. II. Effect of superficial tissue thickness on the sensitivity of the near-infrared spectroscopy signal" Appl. Optics 42, 2915-2922 (2003).

[26] S. Umeyama and T. Yamada, "Monte Carlo study of global interference cancellation by multidistance measurement of near-infrared spectroscopy" J. Biomed. Opt. 14, 064025 (2009).

[27] N. Okui and E. Okada, "Wavelength dependence of crosstalk in dual wavelength measurement of oxy- and deoxy-hemoglobin" J. Biomed. Opt. 10, 011015 (2005).

[28] T. Yamada, S. Umeyama, and K. Matsuda, "Multidistance probe arrangement to eliminate artifacts in functional near-infrared spectroscopy" J. Biomed. Opt. 14, 064034 (2009).

[29] S. J. Matcher, C. E. Elwell, C. E. Cooper, M. Cope, and D. T. Delpy, "Performance comparison of several published tissue near-infrared spectroscopy algorithms" Anal. Biochem. 227, 54-68 (1995).

[30] M. S. Cohen, "Real-time functional magnetic resonance imaging" Methods 25, 201-220 (2001).

[31] C. E. Elwell, R. Springett, E. Hillman, and D. T. Delpy, “Oscillations in cerebral haemodynamics. Implications for functional activation studies" Adv. Exp. Med. Biol. 471, 57-65 (1999).

[32] T. Müller, J. Timmer, M. Reinhard, E. Oehm, and A. Hetzel, "Detection of very low-frequency oscillations of cerebral haemodynamics is influenced by data detrending" Med. Biol. Eng. Comput. 41, 6974 (2003).

[33] F. Scholkmann, S. Spichtig, T. Muehlemann, and M. Wolf, "How to detect and reduce movement artifacts in near-infrared imaging using moving standard deviation and spline interpolation" Physiol. Meas. 31, 649-662 (2010).

[34] Y. Yamashita, A. Maki, and H. Koizumi, "Wavelength dependence of the precision of noninvasive optical measurement of oxydeoxy-, and total-hemoglobin concentration" Med. Phys. 28, 11081114 (2001).

[35] S.S. Long, T. B. Zhang, and F. Long, "Causes and solutions of overshoot and undershoot and end swing in Hilbert-Huang transform" Acta Seismologica Sinica 18, 602-610 (2005).

[36] S. R. Qin and Y. M. Zhong, "A new envelope algorithm of HilbertHuang Transform" Mech. Syst. Signal Pr. 20, 1941-1952 (2006).
[37] Z. P. Fan, T. S. Hong, Z. Z. Liu, and Z. Z. Jing, "Improve the Envelope of EMD with Piecewise Linear Fractal Interpolation" Key Eng. Mat. 439-440, 390-395 (2010).

[38] H. Li, H. H. Hao, and Y.L. Sun, "Improved algorithm for empirical mode decomposition with independent elements" J. Harbin Inst. Tech. 41, 245-248 (2009).

[39] L. Zhao, X. F. Liu, S. R. Qin, P. H. Ju, and F. Zhao, "Use of masking signal to improve empirical mode decomposition" J. Vib. Shock 29, 13-17 (2010).

[40] M. A. Franceschini, S. Fantini, J. H. Thompson, J. P. Culver, and D. A. Boas, "Hemodynamic evoked response of the sensorimotor cortex measured noninvasively with near-infrared optical imaging" Psychophysiology 40, 548-560 (2003).

[41] M. A. Franceschini, D. K. Joseph, T. J. Huppert, S. G. Diamond, and D. A. Boas, "Diffuse optical imaging of the whole head" J. Biomed. Opt. 11, 054007 (2006). 